Part of Journal of Research of the National Bureau of Standards, Volume 20, March 1938

\title{
PHOTOELASTIC DETERMINATION OF STRESSES AROUND A CIRCULAR INCLUSION IN RUBBER
}

\author{
By Wilfred E. Thibodeau and Lawrence A. Wood
}

\begin{abstract}
Analysis is made of the stresses around a rigid circular inclusion with cemented boundary in a rubber sheet which is under simple tension at a distance from the inclusion. The analysis is performed by the photoelastic method, in which data are obtained from observations of the changes produced in a beam of polarized light passing through the transparent model. The fundamental principles of the method and a detailed account of their application are given. The possibilities of the use of rubber as a model material are demonstrated. The stress-optical coefficient of a certain soft vulcanized rubber compound is found to be 2,030 brewsters. This is in agreement with the results of previous work. The maximum stresses near the boundary of the disk are found to be about 50 percent greater than the average applied stress. The experimental results are compared with those given by a theoretical treatment of this problem, and quite satisfactory agreement is obtained.
\end{abstract}

\section{CONTENTS}

Page

I. Introduction

II. Theory of stresses_._.

1. Principal stresses

2. Isoclinic lines _

3. Isostatic lines... 395

4. Maxwell's equation of equilibrium 396

III. Photoelastic theory _... 398

1. Stress-optical coefficient

2. Location of isoclinic lines

IV. Experimental arrangement.

1. Apparatus

2. Model .

V. Procedure _........... 400

1. Determination of stress-optical coefficient $\ldots \ldots 0$

2. Location of isoclinic lines. 402

3. Location of isostatic lines

4. Evaluation of stresses. 402

VI. Theoretical solution

VII. Discussion of results

VIII. Conclusions _.

\section{INTRODUCTION}

The problem of the stress distribution around a rigid inclusion in a body under tension is of considerable interest in a number of different fields, and yet there has been comparatively little work done on it. The related problem of the stresses around a hole has received much more attention. In the case of rubber, the problem of the rigid inclusion is particularly important not only with respect to inclusions of appreciable size, but also with respect to the microscopic and sub- 
microscopic filler particles, which must give rise to some rather complex stress distributions. The present investigation of the stresses around a circular inclusion in rubber was originally undertaken with the aim of solving the much more complicated problem of the distribution of stress in rubber containing filler particles. Some preliminary work was done, on the mutual effect of the stresses arising from the presence of more than one inclusion, but the investigation was not completed.

For the experimental solution of such stress problems the extremely useful method of photoelastic analysis has come into prominence in recent years, especially since the publication by Coker and Filon of a comprehensive treatise ${ }^{1}$ on the subject. The necessary data are obtained by observing in polarized light the patterns obtained when a transparent model is stressed.

Many years ago Maxwell ${ }^{2}$ outlined the theory underlying the general methods of such analysis, but it remained for Mesnager, ${ }^{3}$ Coker, ${ }^{4}$ and others to develop practical procedure ${ }^{56}$ and the materials ${ }^{7}$ for the models. Extensive use of the method has been made in the study of engineering materials with models constructed of glass, Celluloid, Bakelite, and other substances, especially the organic plastics.

Since the results of a previous investigation ${ }^{8}$ of the photoelastic properties of soft vulcanized rubber have indicated that some rubber compounds fulfill many of the requirements for a desirable model material, it was decided to study the problem of the stresses around a rigid circular inclusion with a cemented boundary in a rectangular model consisting of a sheet of transparent, soft vulcanized rubber under tension in one direction. In this manner, therefore, contrary to the usual practice, the problem could be studied with a model of the very material for which the solution was desired. Furthermore, by a definite illustration, it was desired to give a demonstration of the possibilities of the use of rubber as a model material in photoelastic work, and to present in convenient form for those unfamiliar with this field a detailed outline and illustration of the exact experimental procedure used for the solution of problems by this method.

\section{THEORY OF STRESSES}

\section{PRINCIPAL STTRESSES}

Through every point in a plane section under stress there is a pair of mutually perpendicular lines across which the stresses are normal, the shearing stresses being zero in this case alone. These normal stresses are known as the principal stresses at this point. Across all other lines through the point, in general, shearing stresses exist. The directions of the principal stresses, if joined in continuous curves throughout the body, form a system of mutually orthogonal lines.

\footnotetext{
1 E. G. Coker and L. N. G. Filon. A Treatise on Photoelasticity (Cambridge Univ. Press, 1931).

2 J. C. Maxwell. On the equilibrium of elastic solids. Trans. Roy. Soc. Edinburgh 20, 87 (1853); Reprinted in Scientific Papers 1, 30 (Cambridge Univ. Press, 1890).

3 A. Mesnager. Contribution to the study of the elastic deformation of solids. Ann. Ponts et Chaussees 4 , 128 (1901); Determination of stresses in a glass model. 16, 133 (1913).

E. G. Coker. Photoelasticity for engineers. Gen. Elec. Rev. 23, 870 (1920); 23, 966 (1920); 24, 82 (1921); 24, 222 (1921); $\mathbf{2 4}, 455$ (1921).

'A. L. Kimball. Optical determination of stress in a transparent material. J. Opt. Soc. Am. 5, 279 (1921)

H. B. Maris. Photoelastic investigations. J. Opt. Soc. Am. 15, 203 (1927).

R. B. Carleton. Suitability of materials for photoelastic investigations. Rev. Sci. Instr. 5, 30 (1934)

- Wilfred E. Thibodeau and Archibald T. McPherson. Photoelastic properties of soft, vulcanized rubber J. Research NBS 13, 887 (1934) RP751. Reprinted in Rubber Chem. Tech. 8, 183 (1935).
} 
Knowledge of the magnitude and direction of the principal stresses at every point in the body furnishes a complete solution of the stress problem, both the normal and shearing stresses in any other direction being derivable by means of simple equations.

The symbols $P$ and $Q$ will be used to represent the maximum and minimum principal stresses, respectively, at a point in the plane of stress. Since neither normal nor shearing stresses can exist on a free boundary, the value of one of the principal stresses at a free boundary is zero. Tensile stresses will be represented by positive values for $P$ or $Q$, while compressive stresses will be represented by negative values.

\section{ISOCLINIC LINES}

As an aid to the expression of the directions of the principal stresses at all points in a plane section under stress, it is convenient to define a family of curves, called isoclinic lines, along each of which the direction of the principal stress $P$ makes a constant angle with the direction of applied stress. This constant angle, the parameter of the isoclinic, here denoted by $\phi$, is the same as that between the normal to the direction of the applied stress and the direction of the $Q$ stress. The family of isoclinic lines, then, serves to specify completely the directions

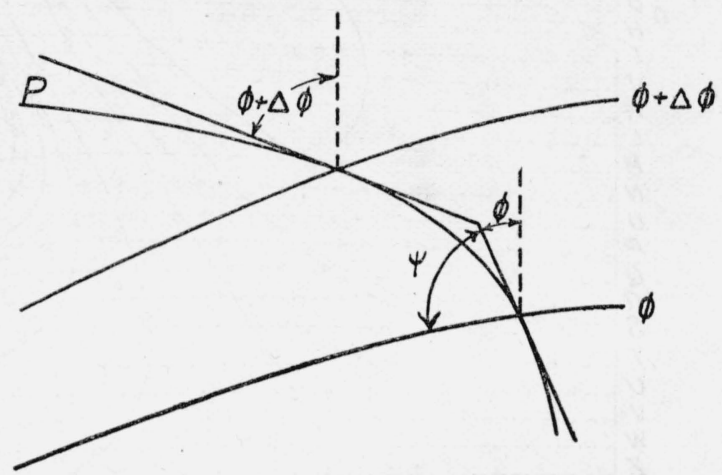

FIGURE 1.--Method of plotting isostatic lines from the isoclinic lines.

of the principal stresses at all points in the plane section. It will be shown later that the isoclinics in a transparent medium under stress may become visible in polarized light under the proper conditions, and can be traced from direct observation.

\section{ISOSTATIC LINES}

Another family of curves, called isostatic lines or simply lines of stress, can be drawn so as to indicate by their direction at any point, the direction of one of the principal stresses. In other words, for example, the direction of the $Q$ stress at any point is given by the tangent to the $Q$ isostatic at that point.

This manner of plotting the isostatics from the isoclinics is shown in figure 1. Let $\phi$ and $\phi+\Delta \phi$ be adjacent isoclinics. $\phi$ then is the locus of all points at which the principal stress line makes an angle $\phi$ with the zero position, and at the points along $\phi+\Delta \phi$ the principal stresses are inclined at the angle $\phi+\Delta \phi$. If now at certain intervals along $\phi$, lines are drawn making the angle $\phi$ with the zero position, these will be tangent to the principal stress lines. These lines are continued to a point midway between the two isoclinics, and from there are continued through $\phi+\Delta \phi$ at an angle $\phi+\Delta \phi$. A continuation of 
this process will furnish an envelope to which the principal stress lines can be drawn. After one set of principal stress lines has been completed in this manner, the other family of stress lines can be drawn in at right angles to the first or it can be obtained from the isoclinics in the same manner as the first.

The isoclinics and one set of isostatics, the $Q$ lines, for the present case are shown in figure 2. These lines in themselves are not sufficient for a complete solution of the stress problem, since this requires the magnitudes of $P$ and $Q$ as well as their directions.

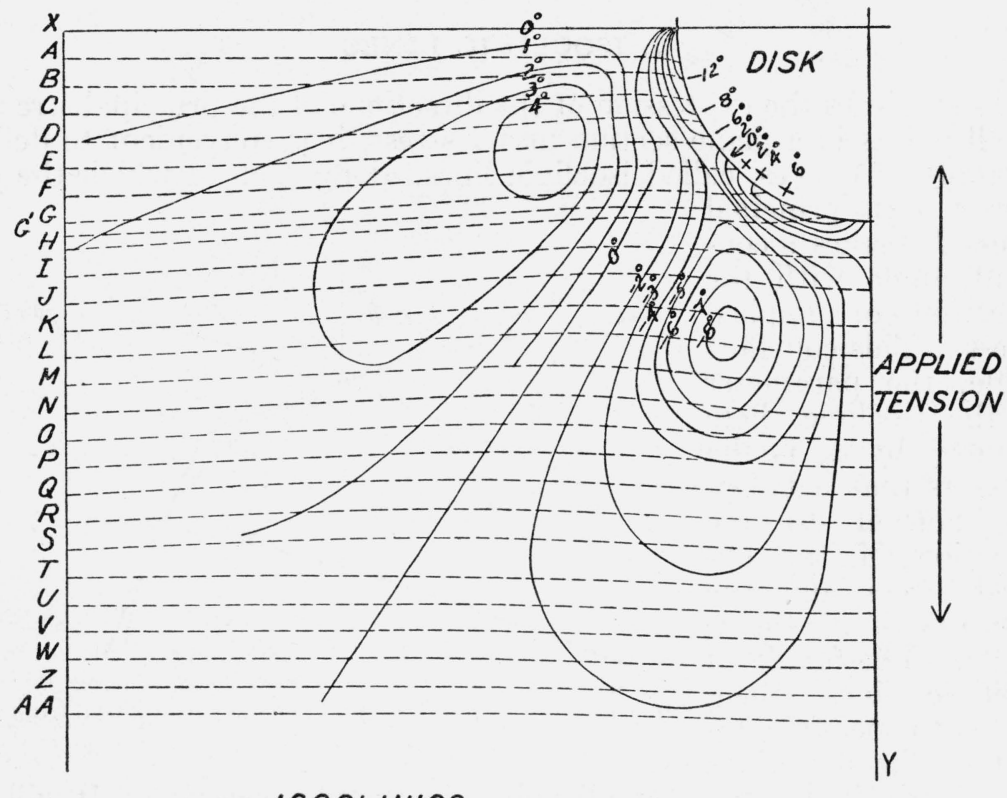

\section{ISOCLINICS}

ISOSTATICS

FIGURE 2.-Isoclinic and $O$-isostatic lines.

\section{MAXWELL'S EQUATION OF EQUILIBRIUM}

Maxwell, in his fundamental work, presented a pair of simplified equations of equilibrium: ${ }^{9}$

$$
\begin{aligned}
& \frac{P-Q}{r_{\mathrm{Q}}}+\frac{\partial P}{\partial s_{\mathrm{p}}}=0 \\
& \frac{P-Q}{r_{\mathrm{P}}}-\frac{\partial Q}{\partial s_{\mathrm{Q}}}=0
\end{aligned}
$$

On integration eq 1a yields:

$$
P=P_{0}-\int_{0}^{S_{\mathrm{p}}} \frac{P-Q}{r_{\mathrm{Q}}} d s_{\mathrm{p}}
$$

? J. C. Maxwell. On the equilibrium of elastic solids. Trans. Roy. Soc. Edinburgh 20, 87 (1853); Reprinted in Scientific Papers I, 30 (Cambridge Univ. Press, 1890). For a derivation of these equations, see page 129 of S. Timoshenko, Theory of Elasticity (McGraw-Hill Book Co., New York, N. Y., 1934). 
in which $P$ and $Q$ are the principal stresses, $s_{\mathrm{P}}$ is the distance along the $P$ line from the point where $P=P_{0}, S_{\mathrm{p}}$ is the value of $s_{\mathrm{p}}$ at the point considered, and $r_{\mathrm{a}}$ is the radius of curvature of the $Q$ line at this point. Similarly, integration of eq $1 \mathrm{~b}$ gives:

$$
Q=Q_{0}+\int_{0}^{S_{Q}} \frac{P-Q}{r_{\mathrm{P}}} d s_{\mathrm{Q}}
$$

in which $s_{0}$ is the distance along the $Q$ line from the point where $Q=Q_{0}, S_{Q}$ is the value of $s_{Q}$ at the point considered, and $r_{\mathrm{P}}$ is the radius of curvature of the $P$ line at this point. $P_{0}$ and $Q_{0}$, the constants of integration, must be separately determined.

It is to be noted that these equations are merely equations of equilibrium, and so involve no elastic constants. Hence they will apply as well to plastic as to elastic substances; and this includes substances which have been stretched beyond the elastic limit and materials like rubber, which do not conform to Hooke's law. The only restrictions are that the material be homogeneous and in a state of equilibrium.

In practical work with a plot of isoclinics and isostatics it is somewhat difficult to measure directly the values of $r_{\mathrm{Q}}$ and $r_{\mathrm{p}}$, and it is easier to compute them from the relation $r=\operatorname{arc} /$ angle $=\Delta S / \Delta \phi$, where $\Delta S$ is the arc on the stress line normal to the path of integration and bounded by the isoclinies of parameters $\phi$ and $\phi+\Delta \phi$ in the neighborhood of the point.

Furthermore, there are a number of advantages of improved applicability in expressing results in terms of ratios of the principal stresses to some applied stress rather than in terms of the principal stresses themselves. In the first place, the results are then independent of the stress units employed. Secondly, the results are immediately applicable to cases in which the applied load is different from that which happened to be employed in the particular case under consideration. Finally, a comparison with the results of a theoretical treatment is possible at each point of the sheet in terms of merely the coordinates of the point.

Therefore, eq 2 and 3 are modified by a substitution for $r$ and by dividing both sides by the applied stress $T$, defined in the present case as the ratio of the applied load to the reduced cross-sectional area of the model.

$$
\begin{aligned}
& \frac{P}{T}=\frac{P_{0}}{T}-\int_{0}^{S_{P}} \frac{P-Q}{T} \frac{\Delta \phi}{\Delta s_{Q}} d s_{P} \\
& \frac{Q}{T}=\frac{Q_{0}}{T}+\int_{0}^{S_{Q}} \frac{P-Q}{T} \frac{\Delta \phi}{\Delta s_{P}} d s_{Q}
\end{aligned}
$$

An alternative modification of eq 2 and 3, suggested by Filon ${ }^{10}$ gives:

$$
\begin{aligned}
& \frac{P}{T}=\frac{P_{0}}{T}+\int_{\phi_{0}}^{\phi} \frac{P-Q}{T} \cot \psi_{P} d \phi \\
& \frac{Q}{T}=\frac{Q_{0}}{T}-\int_{\phi_{0}}^{\phi} \frac{P-Q}{T} \cot \psi_{Q} d \phi
\end{aligned}
$$

${ }^{10} \mathrm{~L}$. N. G. Filon. Graphical determination of stress from photoelastic observations. Engineering 116, 511 (1923); Reprinted in Brit. Assn. Rep. 91, 350 (1923). 
Here $\psi_{P}$ and $\psi_{Q}$ represent the angles through which the $P$ line of stress and the $Q$ line of stress, respectively, must be rotated in a counterclockwise direction to bring them upon the isoclinic, and $\phi_{0}$ is the value of $\phi$, where $P=P_{0}$ and $Q=Q_{0}$.

The photoelastic method, as shown below, makes possible the evaluation of the quantities either in eq 4 and 5 or in eq 6 and 7 . The question of which method to use is a matter of judgment and depends on the configuration of isoclinics and isostatics at the point considered. The ideal case for eq 4 and 5 is where $\psi=0$, that is, where the line of stress is itself an isoclinic. The ideal case for eq 6 and 7 is that in which the isoclinics are inclined at an angle of about $45^{\circ}$ to the lines of stress and are closely packed. If $\psi$ is too small the errors will be large in this method. In the present work both methods were used, sometimes even along the same line of integration.

\section{PHOTOELASTIC THEORY}

\section{STRESS-OPTICAL COEFFICIENT}

When light passes at normal incidence through a sheet of isotropic transparent material stressed in its plane, it emerges, as in the case of doubly-refracting uniaxial crystals, as two plane-polarized rays called ordinary and extraordinary rays, one polarized in the plane of one of the principal stresses and the other polarized at right angles to it, in the plane of the other principal stress. The velocities of these two rays are different, and depend on the intermolecular and interatomic forces in the directions of the principal stresses. The velocity of the ordinary ray is found to be proportional to the $P$ stress plus a constant, and the velocity of the extraordinary ray to vary in exactly the same way with $Q$. The difference of the velocities is then proportional to the difference of principal stresses. The difference in the velocities of the two rays introduces a phase difference as they emerge from the medium. The phase change in each ray, as it passes through the medium, is proportional to the thickness of the medium and inversely proportional to the velocity of that ray. The phase difference, or retardation of one with respect to the other, is then proportional to the thickness and to the difference of the reciprocals of the velocities. Since the difference in the two velocities is extremely small compared with the velocities themselves, the difference of the reciprocals is approximately proportional to the difference of the velocities themselves.

It can thus be seen that the retardation of the ordinary with respect to the extraordinary ray at any point in the body is proportional to the difference of the principal stresses $P$ and $Q$ and to the thickness of the sample. That is,

$$
R=C(P-Q) t,
$$

where $R$ is the relative retardation, $t$ is the thickness of the medium, and $C$, thus defined, is a constant known as the stress-optical coefficient. ${ }^{11}$ When the relative retardation is expressed in angstrom units, the stress in bars ( 1 bar $=10^{6}$ dynes $/ \mathrm{cm}^{2}=1.0197 \mathrm{~kg} / \mathrm{cm}^{2}=14.504$ $\mathrm{lb} /$ in. $\left.^{2}\right)$, and the thickness in millimeters, the stress-optical coefficient is given in brewsters. A brewster is thus $10^{-13} \mathrm{~cm}^{2} /$ dyne.

\footnotetext{
11 E. G. Coker and L. N. G. Filon. A Treatise on Photoelasticity, p. 185 (Cambridge University Press, 1931).
} 
When the value of $C$ for the material is known, the value of $(P-Q)$ at any point in the medium can be obtained by a determination of the retardation and a measurement of the thickness. This law, as expressed in equation 8 , has been fairly definitely established for materials like Bakelite, Celluloid, and glass, and more recently for some vulcanized rubber compounds. ${ }^{12}$ A compound (compound $B$, composition given below) which conformed to this relation was selected for the present investigation.

\section{LOCATION OF ISOCLINIC LINES}

It will now be shown how, as previously stated, in polarized light under the proper conditions the isoclinic lines become visible and can be traced from direct observation. Light which has been polarized by passage through a nicol prism, called the polarizer, passes through the sheet and is observed through a second nicol prism, the analyzer. At all points where the vibration plane of the polarizing nicol is at right angles to the direction of one of the principal stresses, one of the usual two plane-polarized rays passing through the medium is absent, since there is then no component of vibration corresponding to the plane of vibration of this ray. Under the same conditions if the analyzing nicol is set at right angles to the polarizing nicol, the second planepolarized ray, which is actually present in the specimen, is not observed since it can not pass the second nicol. That is, as far as the point in question is concerned, the result is the same as if the model were not interposed. Hence a dark band or brush will appear marking the locus of all points in the model where the principal stress directions correspond with the vibration directions of the nicol prisms. In order to obtain a complete map of the isoclinics it is necessary merely to rotate the nicols by stages through $90^{\circ}$, keeping their vibration planes at right angles and tracing out one resulting isoclinic at each setting. The orientation of the prisms at each position is the parameter of the resulting isoclinic.

\section{EXPERIMENTAL ARRANGEMENT}

\section{APPARATUS}

The apparatus used for the photoelastic determinations consisted essentially of a light source, two nicol prisms, means for supporting and stressing the model, suitable lenses, and a screen. The light source was a 1,000-watt projection lamp, with a water filter for the reduction of heating effects. By means of condensing lenses the light rays were brought to a focus within the nicol prisms and were reduced to parallelism in the region between the nicols where the model was placed. A further refinement, the placing of divergent lenses on either side of the nicol prisms so as to transmit the rays through them in a parallel beam, was found to be unnecessary with the large nicol prisms used. Both prisms were equipped with divided circular scales so that their inclinations could be recorded.

An arrangement of two displaceable quartz wedges known as the Babinet compensator was inserted between the model and the analyzing nicol when it was desired to measure the retardation produced in

12 Wilfred E. Thibodeau and Archibald T. McPherson. Photoelastic properties of soft, vulcanized rubber. J. Research NBS 13, 887 (1934) RP751. Reprinted in Rubber Chem. Tech. 8, 183 (1935). 
the stressed model. The compensator itself produced a retardation in the opposite direction amounting to 11,400 angstrom units for each centimeter of displacement of the wedges. In this manner the net retardation could be reduced to zero, and measurement made of the amount of retardation required to accomplish this. The compensator was mounted so that it could be turned until its axes were parallel to the principal stresses at the point under observation. The final lens focused an image of the model on a piece of plain white paper, which served adequately as a screen.

\section{MODEL}

The model was a rectangular sheet of soft transparent rubber 46.0 by 29.8 by $0.651 \mathrm{~cm}$, in the center of which was placed a disk of stiff rubber-flooring material, the moduli of which were very great in comparison with those of the soft rubber. The disk was $5.22 \mathrm{~cm}$ in diameter and $0.62 \mathrm{~cm}$ in thickness. The periphery of the disk was coated with rubber cement (Vulcalock), and after vulcanization the disk became firmly embedded in the model.

The composition of the soft rubber was as follows:

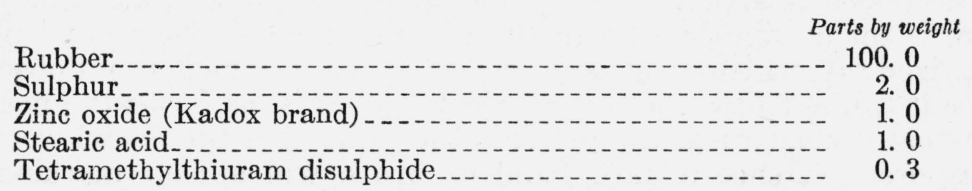

This was cured for 45 minutes at $125^{\circ} \mathrm{C}$ in a steam press. The weight of the model was $858 \mathrm{~g}$. It was loaded vertically by means of wooden clamps bolted to each end of the model. The weight of the lower clamps and bolts was $170 \mathrm{~g}$.

\section{PROCEDURE}

\section{DETERMINATION OF STRESS-OPTICAL COEFFICIENT}

For the purpose of determining the photoelastic characteristics of the rubber, a separate tensile specimen of flat dumbbell shape was prepared from the same compound, and stretched. The results of observations in the central region of uniform stress are shown in table 1. Since here $Q=0$ and $P=T$, by the application of eq 8 to this case, one finds:

$$
R=C T t,
$$

where $T$ is the applied stress, measured on the actual cross section and $t$ the actual thickness. If $\sigma$ is the compensator constant, the retardation per unit displacement of the compensator, and $X$ the displacement,

$$
\begin{aligned}
R & =\sigma X=C T t \\
T & =\frac{\sigma}{C t} X
\end{aligned}
$$


TABLE 1.-Data for calculation of stress-optical coefficient

[Original thickness $0.278 \mathrm{~cm}$; original width $0.632 \mathrm{~cm}$ ]

$W=$ Applied load .

$L=$ Distance between gage marks.

$X=$ Displacement of compensator.

\begin{tabular}{|c|c|c|}
\hline$W$ & $L$ & $X$ \\
\cline { 1 - 2 } $\mathrm{kg}$ & $\mathrm{cm}$ & $\mathrm{cm}$ \\
0.000 & 4.98 & 0.000 \\
.055 & 5.13 & .145 \\
.123 & 5.31 & .330 \\
.173 & 5.46 & .485 \\
.223 & 5.62 & .650 \\
.273 & 5.77 & .815 \\
.323 & 5.98 & .970 \\
.373 & 6.18 & 1.140 \\
.423 & 6.40 & 1.345 \\
.473 & 6.63 & 1.510 \\
\hline
\end{tabular}

For pure gum rubber vulcanizate, which was used here, Poisson's ratio has been found ${ }^{13}{ }^{14}$ to be 0.500 for elongations in the range under consideration. For an isotropic medium with a Poisson's ratio of 0.5 , it may easily be shown that

and

$$
T=T_{0}(1+\epsilon)
$$

$$
t=\frac{t_{0}}{\sqrt{1+\epsilon}}
$$

where $T_{0}$ is the applied stress calculated on the original cross section, $t_{0}$ the original thickness, and $\epsilon$ the strain, or fractional elongation. Then

$$
T_{0}(1+\epsilon)=\frac{\sigma^{\circ}}{C t_{0}} X \sqrt{1+\epsilon}
$$

This equation predicts proportionality between the quantity $T_{0}(1+\epsilon)$ and the quantity $X \sqrt{1+\epsilon}$.

A graph of the experimental data of table 1 verifies this proportionality and has a slope ${ }^{15}$ of $2.06 \mathrm{~kg} \mathrm{~cm}^{-3}$. On multiplication of this slope by the thickness, the value of $\sigma / C$ is found to be $0.572 \mathrm{~kg} \mathrm{~cm}^{-2}$. It is not necessary to compute the actual value of $C$.

Turning now to a consideration of the stretched model with the circular insertion, the mean thickness $t_{m}$ of the model being $0.611 \mathrm{~cm}$, one can apply eq 8 to obtain

$$
P-Q=\frac{\sigma}{C t_{m}} X=\frac{0.572}{0.611} X=0.936 X
$$

Since the thickness of the model varied from point to point, strictly the individual values should be used instead of a single mean thickness. This more rigorous method was applied at a number of selected

12 Wilfred E. Thibodeau and Archibald T. McPherson. Photoelastic properties of soft, vulcanized rubber J. Research NBS 13, 887 (1934) RP751. Reprinted in Rubber Chem. Tech. 8, 183 (1935).

${ }_{14}$ William L. Holt and Archibald T. McPherson. Change of volume of rubber on stretching: Effects of time, elongation, and temperature. J. Research NBS 17, 657 (1936) RP936. Reprinted in Rubber Chem. Tech. $10,412(1937)$.

is From this slope a value of the stress-optical coefficient $C$ may be calculated. Here $t_{0}=2.78 \mathrm{~mm}$, and $\sigma=11,400 \mathrm{~A} / \mathrm{cm}$.

$$
C=\frac{11,400}{(2.78)(2.06)(980) 10^{-3}}=2,030 \text { brewsters. }
$$

This result is in reasonable agreement with the value 2,096 brewsters, the average obtained over a much larger range of stresses for seven samples of this compound in the previous investigation by Thibodeau and McPherson (reference 13). 
points, and was found to yield results differing from the simpler method by only about 5 percent at the most. In view of the difficulties of measurement and the increased complexity of calculation, it did not seem worth-while to utilize this more refined procedure.

The load applied near the center of the model included, in addition to an external load of $26.75 \mathrm{~kg}$, the weight of the lower clamps and half the weight of the model. When this total load of $27.35 \mathrm{~kg}$ was applied, the distance between the clamps increased from 41.2 to 46.1 $\mathrm{cm}$, the width of the model at the center decreased from 29.8 to 28.3 $\mathrm{cm}$, and its mean thickness decreased from 0.651 to $0.611 \mathrm{~cm}$. Dividing $27.35 \mathrm{~kg}$ by $17.3 \mathrm{~cm}^{2}$, the reduced cross-sectional area, one obtains $1.58 \mathrm{~kg} / \mathrm{cm}^{2}$ as the value of $T$, the applied stress. Finally,

$$
\frac{P-Q}{T}=\frac{0.936}{1.58} X=0.593 X
$$

This is the relation employed in the calculations involving the data obtained with the compensator.

\section{LOCATION OF ISOCLINIC LINES}

With the plane of vibration of the polarizing nicol vertical and the plane of vibration of the analyzing nicol horizontal, the external load of $26.75 \mathrm{~kg}$ was applied to the model along the vertical direction. The isoclinic with parameter $\phi=0^{\circ}$ appeared as a dark line on the screen and was traced on the paper. Both nicols were then rotated $1^{\circ}$ and the corresponding isoclinic was traced. This process was repeated until the complete pattern of isoclinics shown in figure 2 was obtained.

\section{LOCATION OF ISOSTATIC LINES}

When the complete family of isoclinics had been traced, the paper was removed and the $Q$ isostatics shown in figure 2 were drawn in by the method already outlined and illustrated in figure 1.

\section{EVALUATION OF STRESSES}

The paper screen was again inserted in the optical system, and the Babinet compensator was introduced between the model and the analyzing nicol. For each determination the axes of the compensator were turned so as to be parallel with the lines of principal stress at the point considered. The nicol prisms, ' with their axes still perpendicular to each other, were turned so that the axes made positive and negative angles of $45^{\circ}$ with the vertical. At every point where an isostatic was crossed by an isoclinic and at other suitable points, measurements were made of the compensator displacement necessary to nullify the retardation produced in the model. From these values, which are proportional to the differences of principal stresses, as bas just been shown, the magnitudes of $(P-Q) / T$ were determined by the use of eq 15.

In all this work, when the load was removed the rubber showed no evidence of double refraction even after repeated cycles of stress. This fact proved the absence of initial residual stresses, the presence of which is one of the commonest faults of most model materials.

Sufficient data were now available for the evaluation of the principal stresses by the use of one or more of the eq 4, 5, 6, and 7 . For example, in the data which were taken along the $x$-axis and which are given in table 2 , the isostatic is itself an isoclinic, and, as already stated, this 
is the ideal case for the application of eq 5 . The logical place to begin the integration is at a free boundary, where the $Q$ stress is known to be zero. Unfortunately, in the present instance no observation was made at the boundary. Therefore, the value -0.055 , obtained by the theoretical solution for an infinite sheet as given in the section below, was taken for $Q_{0} / T$ at the first point of observation. The magnitude of such an assumed value is likely to be slightly too large because of the finite size of the sheet.

At successive points along the isostatic, four observations were made: (1) $S_{\alpha}$, the distance along the $Q$ stress line from the point of commencement of the integration to the point considered; (2) $\Delta S_{\mathrm{p}}$, the distance, measured along the $P$ stress line through the point, between the two nearest isoclinics lying on opposite sides of the $Q$

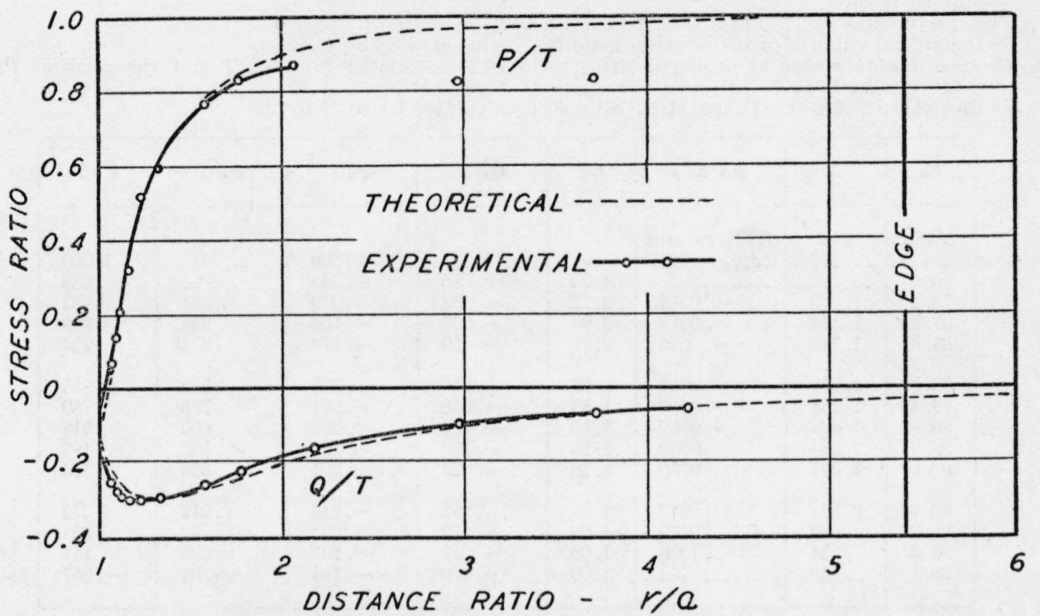

Figure 3.-Principal stresses along the $x$-axis.

Later independent observations failed to indicate as large a discrepancy between experimental and theoretical values as is indicated for the values of $P / T$ at $r / a=2.97$ and $r / a=3.72$. Therefore, the experimental curve has not been continued through these points.

line and approximately equidistant from it, this approximate equality being brought about, if necessary, by the proper selection of the point to be considered; (3) $\Delta \phi$, the amount in radians by which these isoclinics differ; and (4) $(P-Q) / T$ from the compensator reading.

Graphic evaluation of the integral in eq 5 could be made by measurement of the area under the curve whose coordinates were $\left[\left(\frac{P-Q}{T}\right) \frac{\Delta \phi}{\Delta S_{\mathrm{p}}}\right]$ and $S_{\mathrm{q}}$. These curves were not actually drawn in the present work, but the value of the integral was obtained for each interval as the product of mid-ordinate by the interval length.

The addition of values obtained in this manner yielded the results given for $Q_{e} / T$ in table 2 and in figure 3. Finally, computations were made from the relation $P / T=(P-Q) / T+Q / T$ to obtain the results shown for $P_{e} / T$.

Along the $y$-axis, where again the isostatic is itself an isoclinic, there occurs the ideal case for the application of eq 4 . The operation of obtaining $P / T$ here was carried out in quite similar fashion to what has just been described in the application of eq 5 . The value of $P_{0} / T$ was taken from the theoretical solution as 1.054 . The results 
are shown in table 3 in the column headed $P_{e} / T$. Values of $Q / T$ obtained from the relation $Q / T=P / T-(P-Q) / T$ are given in table 3 in the column headed $Q_{e} / T$. Since these values are obtained as the differences of two quantities which are much larger than these differences, the accuracy would not be expected to be so good as if they were obtained more directly.

\section{TABLE 2.-Principal stresses along the $x$-axis}

$S_{Q}=$ Distance along the $Q$ isostatic, measured in arbitrary units of such size that the radius of the disk $(a=2.61 \mathrm{~cm})$ is equal to 30 units.

$S_{P}=$ Distance along the $P$ isostatic, measured in the same units as $S_{Q}$. Since eq 5 and 6 involve merely the ratio of these quantities, the size of the unit is immaterial.

$X=$ compensator displacement.

$\phi=$ parameter of the isoclinic, namely, the angle from the direction of the applied tension to the direction of the $P$ stress.

$r=$ distance from the center of the disk.

$a=$ radius of the disk.

$T=$ applied tension.

Q. $T=$ experimental value of principal stress ratio $Q / T$, calculated by eq 5 .

$Q_{t} \mid T=$ theoretical value of principal stress ratio $Q / T$, calculated by eq 17 to 22 .

$P . \mid T=$ experimental value of principal stress ratio $P / T$, calculated from $Q_{e} / T$ and the relation $P / T=$ $(P-Q) / T+Q / T$

$P_{t} / T=$ theoretical value of principal stress ratio $P / T$, calculated by eq 17 to 22 .

\begin{tabular}{|c|c|c|c|c|c|c|c|}
\hline$S_{a}$ & $X$ & $\Delta \phi / \Delta S_{P}$ & $r / a$ & $Q_{\bullet} / T$ & $Q_{t} / T$ & $P . / T$ & $P_{t} / T$ \\
\hline Units & $\mathrm{cm}$ & Radians/unit & & & & & \\
\hline n...... & $-\ldots-1$ & hon & $\infty$ & & 0.000 & & 1.000 \\
\hline 0.0 & & 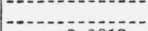 & 4. 23 & $a-0.055$ & -.055 & & .988 \\
\hline 15.2 & 1. 509 & -0.0013 & 3.72 & -.064 & -.070 & 0.831 & .983 \\
\hline 37.8 & 1.554 & -.0018 & 2.97 & -.096 & -.106 & .826 & .969 \\
\hline 61.8 & 1. 749 & -.0035 & 2.17 & -.159 & -.179 & .878 & .924 \\
\hline 73.8 & 1. 779 & -.0056 & 1. 77 & -.216 & -.238 & .839 & .855 \\
\hline 79.5 & 1. 734 & -.0060 & 1.58 & -.250 & -.261 & .778 & .780 \\
\hline 86.8 & 1. 499 & -.0050 & 1. 33 & -.289 & -.297 & .600 & .618 \\
\hline 89.9 & 1. 384 & .0000 & 1. 23 & -.296 & -.290 & .525 & .497 \\
\hline 92.0 & 1. 044 & .0050 & 1.16 & -.293 & -.271 & .326 & .379 \\
\hline 93.5 & 0.834 & .0233 & 1. 11 & -.282 & -.246 & .212 & .272 \\
\hline 94.0 & .699 & .0700 & 1.09 & -.272 & -.233 & .142 & .224 \\
\hline 95.0 & .534 & .1050 & 1. 06 & -.241 & -.207 & .076 & .141 \\
\hline 96.8 & .419 & - & 1. 00 & $b-.170$ & -.133 & $b-.04$ & -.067 \\
\hline
\end{tabular}

- Theoretical value.

Extrapolated value.

For the purpose of obtaining values of the principal stresses around the circumference of the disk and for obtaining independent values of $Q / T$ on the $y$-axis, integrations were carried out along most of the $Q$ isostatics shown in figure 2. Along lines $A$ to $G$ eq 5 was used for about the first six points and eq 7 for the remaining points. For line $G^{\prime}$ and all those below it eq 7 alone was employed. For those points where eq 5 was used, the method of calculation was identical with that performed along the $x$-axis.

With eq 7 three observations were made: (1) $\psi_{Q}$, the angle through which the line of stress must be rotated in a counterclockwise direction to bring it upon the isoclinic, the value being obtained most accurately by measuring the angle between the isoclinic and the horizontal, and subtracting from this angle the parameter of the isoclinic; $(2) \phi$, the parameter of the isoclinic; (3) $(P-Q) / T$ from the compensator reading. The integrations here also were carried out by the multiplication of mid-ordinates and interval lengths. In all these integrations, except along the $x$-axis, the value of $Q_{0} / T$ was taken as zero. $P / T$ was calculated in the same manner as was done along the $x$-axis. Some of the results obtained at the last points of each integration are given in table 4 in columns headed $Q_{e} / T$ and $P_{e} / T$, and the others in the columns headed $Q_{e i} / T$ and $P_{e i} / T$ in table 3. 


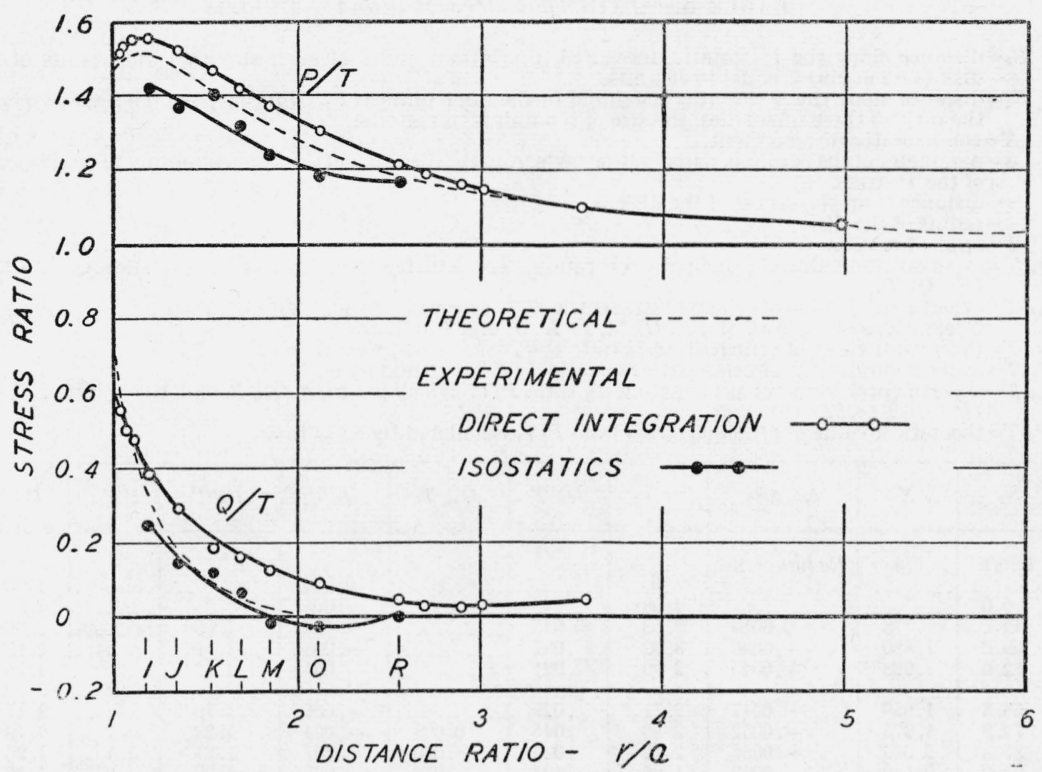

Figure 4.-Principal stresses along the y-axis.

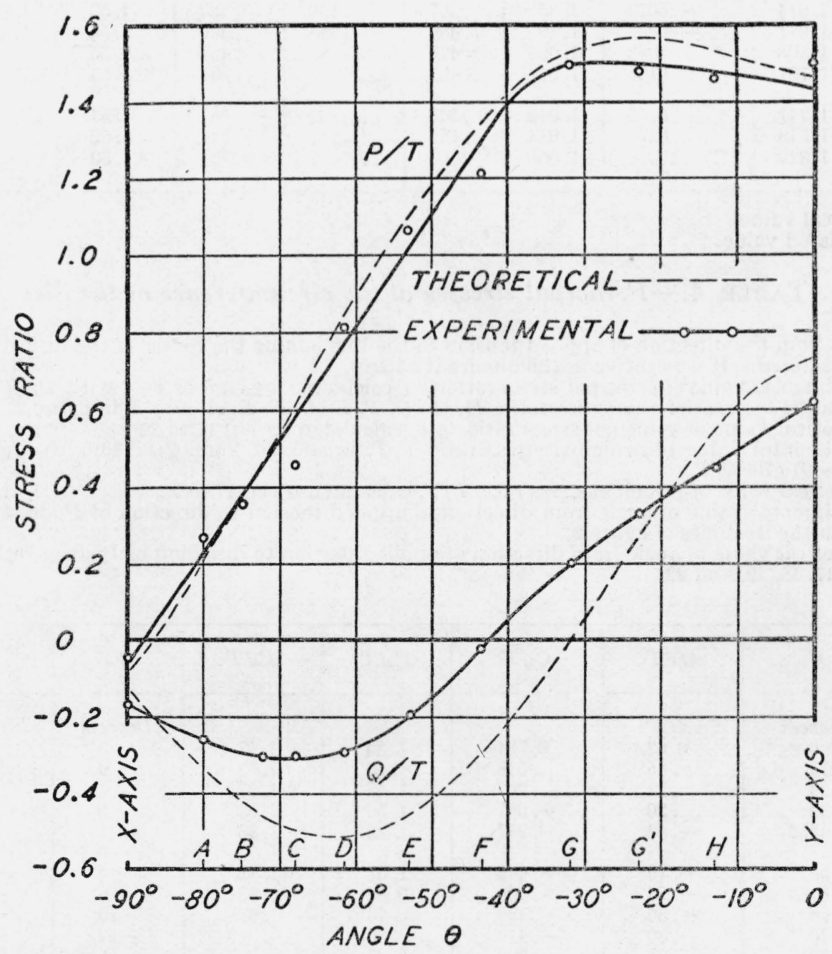

FiguRe 5.-Principal stresses at the circumference of the disk. 


\section{TABLE 3.-Principal stresses along the $y$-axis}

$S_{\mathbf{P}}=$ distance along the $P$ isostatic, measured in arbitrary units of such size that the radius of the disk $(\mathrm{a}=2.61 \mathrm{~cm})$ is equal to 30 units.

$S_{\mathbf{Q}}=$ distance along the $Q$ isostatic, measured in the same units as $S_{\mathbf{P}}$. Since eq 5 and 6 involve merely the ratio of these quantities, the size of the unit is immaterial.

$\boldsymbol{X}=$ compensator displacement.

$\phi=$ parameter of the isoclinic, namely, the angle from the direction of the applied tension to the direction of the $P$ stress.

$r=$ distance from the center of the disk.

$a=$ radius of the disk.

$T=$ applied tension.

$Q_{0} / T=$ experimental value of principal stress ratio $Q / T$, calculated from $P_{e} / T$ and the relation $Q / T=P / T-$ $(P-Q) / T$.

$Q_{e i} / T=$ experimental value of principal stress ratio $Q / T$, calculated by eq 7 with $Q_{0} / T=0$, and with separate integrations along isostatics $R, O, M, L, K, J$, and $I$ in figure 2

$Q_{t} / T=$ theoretical value of principal stress ratio $Q / T$, calculated by eq 17 to 22 .

$P$ e/ $T=$ experimental value of principal stress ratio $P / T$, calculated by eq 4 .

$P_{\bullet i} / T=$ experimental value of principal stress ratio $P / T$, calculated from $Q_{e i} / T$, and the relation $P / T=$ $(P-Q) / T+Q / T$.

$P_{t} / T=$ theoretical value of principal stress ratio $P / T$, calculated by eq 17 to 22 .

\begin{tabular}{|c|c|c|c|c|c|c|c|c|c|}
\hline$S_{P}$ & $X$ & $\Delta \phi / \Delta S_{Q}$ & $r / a$ & $Q_{0} / T$ & $Q_{e i} / T$ & $Q_{t} / T$ & $P_{\odot} / T$ & $P_{e i} / T$ & $P_{t} / T$ \\
\hline Units & $\mathrm{cm}$ & Radians/unit & & & & & & & \\
\hline 0.0 & & & $\begin{array}{l}\infty \\
4.98\end{array}$ & & & $\begin{array}{r}0.000 \\
-.005\end{array}$ & a 1.054 & & $\begin{array}{l}1.000 \\
1.054\end{array}$ \\
\hline 42.2 & 1.773 & -0.0019 & 3.58 & 0.045 & |- & -.008 & 1. 096 & - & $\begin{array}{l}1.097 \\
1.098\end{array}$ \\
\hline 59.0 & 1.880 & -.0035 & 3.02 & .031 & - & -.008 & 1.146 & - & 1.139 \\
\hline 62.6 & 1. 923 & -.0040 & 2.90 & .021 & & -.007 & 1.16 & & 1.15 \\
\hline 68.3 & 1. 959 & -.0047 & 2.71 & .029 & & -.006 & 1.19 & & 1.17 \\
\hline 72.8 & 1.975 & -.0052 & 2.56 & .045 & 0.003 & -.004 & 1. 22 & 1.17 & 1.19 \\
\hline 85.8 & 2.046 & -.0066 & 2.12 & .095 & -.030 & .007 & 1. 31 & 1. 18 & 1.26 \\
\hline 94.0 & 2.110 & -.0073 & 1.85 & .127 & -.012 & .028 & 1.38 & 1. 24 & 1.32 \\
\hline 98.8 & 2. 126 & -.0080 & 1.69 & .164 & .058 & .052 & 1.42 & 1.32 & 1.37 \\
\hline 103.3 & 2. 169 & -.0083 & 1.54 & .186 & .118 & .089 & 1.47 & 1.41 & 1.42 \\
\hline 109.0 & 2.074 & -.0073 & 1.35 & .297 & .140 & .180 & 1.53 & 1.37 & 1.48 \\
\hline 113.8 & 1. 971 & -.0034 & 1.19 & .389 & .248 & .330 & 1. 56 & 1.42 & 1.52 \\
\hline 116.6 & 1.808 & .0083 & 1.095 & .479 & $-\cdots$ & .487 & 1. 55 & $-\ldots-$ & 1.51 \\
\hline 117.3 & 1.761 & .0165 & 1. 073 & .497 & & .531 & 1.54 & & 1.51 \\
\hline 117.7 & 1.741 & .0159 & 1.060 & .502 & & .564 & 1.54 & & 1.50 \\
\hline 118.5 & 1.618 & .0270 & 1. 033 & 559 & & .644 & 1. 52 & & 1.48 \\
\hline 119.5 & 1.320 & & 1.000 & b. 62 & & .733 & b 1.50 & & 1.47 \\
\hline
\end{tabular}

Theoretical value.

- Extrapolated value.

\section{TABLE 4.-Principal stresses at the circumference of the disk}

$\theta=$ angle from the direction of applied tension to the line joining the center of the disk with the point considered. It is negative in the quadrant chosen.

$Q_{0} / T=$ experimental value of principal stress ratio $Q / T$ calculated by eq 5 or eq 7 with $Q_{0} / T=0$, and with separate integrations along isostatics $H, G^{\prime}, G, F, E, D, C, B, A$, and $\mathrm{X}$ in figure 2.

$Q_{t} / T=$ theoretical value of principal stress ratio $Q / T$, calculated by eq. 17 to 22 .

$P_{e} / T=$ experimental value of principal stress ratio $P / T$, calculated from $Q_{e} / T$ and the relation $P / T=$ $(P-Q) / T+Q / T$.

$P_{t} / T=$ theoretical value of principal stress ratio $P / T$, calculated by eq 17 to 22

$\phi_{0}=$ experimental value of angle from direction of applied tension to direction of $P$ stress, as observed from the isoclinics of figure 2 .

$\phi_{\mathrm{s}}=$ theoretical value of angle from direction of applied tension to direction of $P$ stress, calculated from eq $17,18,19$, and 22 .

\begin{tabular}{|c|c|c|c|c|c|c|}
\hline$\theta$ & $Q_{e} / T$ & $Q_{t} / T$ & $P_{\mathrm{e}} / T$ & $P_{t} / T$ & $\phi$. & $\phi_{t}$ \\
\hline $\begin{array}{c}\text { Degrees } \\
0 \\
-13 \\
-23 \\
-32 \\
-43.5\end{array}$ & $\begin{array}{r}0.62 \\
.45 \\
.33 \\
.20 \\
-.02\end{array}$ & $\begin{array}{r}0.734 \\
.548 \\
.267 \\
-.001 \\
-.287\end{array}$ & $\begin{array}{l}1.50 \\
1.46 \\
1.48 \\
1.50 \\
1.22\end{array}$ & $\begin{array}{l}1.47 \\
1.53 \\
1.57 \\
1.53 \\
1.35\end{array}$ & $\begin{array}{c}\text { Degrees } \\
0 \\
9 \\
7 \\
5 \\
1\end{array}$ & $\begin{array}{c}\text { Degrees } \\
0 \\
10 \\
8 \\
3 \\
-5\end{array}$ \\
\hline $\begin{array}{l}-53 \\
-61.5 \\
-68 \\
-75 \\
-80 \\
-90\end{array}$ & $\begin{array}{l}-.19 \\
-.29 \\
-.30 \\
-.30 \\
-.25 \\
-.17\end{array}$ & $\begin{array}{l}-.449 \\
-.508 \\
-.489 \\
-.419 \\
-.329 \\
-.133\end{array}$ & $\begin{array}{r}1.07 \\
0.82 \\
.46 \\
.36 \\
.27 \\
-.04\end{array}$ & $\begin{array}{l}1.12 \\
0.854 \\
.625 \\
.379 \\
.217 \\
-.066\end{array}$ & $\begin{array}{r}-4 \\
-8 \\
-10 \\
-12 \\
-15 \\
0\end{array}$ & $\begin{array}{r}-12 \\
-19 \\
-24 \\
-30 \\
-33 \\
0\end{array}$ \\
\hline
\end{tabular}




\section{THEORETICAL SOLUTION}

The theory of the problem of the stresses in an infinite plate with a heterogeneous circular insertion has been treated by Sezawa and Nishimura ${ }^{16}$ who give a general solution in terms of the elastic constants of the plate and of the insertion. The constants used are $\lambda$, the plane stress constant ${ }^{17}$ (not the Lamé constant of three-dimensional problems) and $\mu$, the rigidity modulus. ${ }^{18}$ In the present application the insertion is to be considered as completely rigid. Therefore, the elastic constants for it may be taken as equal to each other and infinite. This assumption reduces very materially the complexity of the general equations given by Sezawa and Nishimura. Furthermore, the terms involving the elastic constants of the plate may be expressed as functions of Poisson's ratio $\eta$ by the following equation ${ }^{19}$ from the general theory of elasticity;

$$
\eta=\frac{\lambda}{\lambda+2 \mu}
$$

As previously stated, Poisson's ratio for rubber has been found ${ }^{20}$ to be 0.500 for elongations in the present range.

Under these conditions the general equations of Sezawa and Nishimura reduce to the following:

$$
\begin{aligned}
& \frac{\widehat{r}}{\bar{T}}=\frac{1}{2}\left\{1+\frac{1}{3} \frac{a^{2}}{r^{2}}+\left[1+\frac{12}{5} \frac{a^{2}}{r^{2}}-\frac{9}{5} \frac{a^{4}}{r^{4}}\right] \cos 2 \theta\right\} \\
& \hat{\theta \theta}=\frac{1}{T}\left\{1-\frac{1}{3} \frac{a^{2}}{r^{2}}+\left[1-\frac{9}{5} \frac{a^{4}}{r^{4}}\right] \cos 2 \theta\right\} \\
& \hat{r \theta}=-\frac{1}{2}\left[1-\frac{6}{5} \frac{a^{2}}{r^{2}}+\frac{9}{5} \frac{a^{4}}{r^{4}}\right] \sin 2 \theta
\end{aligned}
$$

Here $\theta$ is the angle between the direction of applied stress $T$ and the line $r$ from the center of the disk to the point under consideration; $a$ is the radius of the disk; $\hat{r} r$ and $\hat{r} \theta$ are, respectively, the radial and tangential stresses across a section normal to $r$, while $\widehat{\theta \theta}$ is the stress normal to a section parallel to $r$.

The general theory of plane stresses yields equations ${ }^{21}$ giving stresses referred to any axes rotated by a given angle with respect to selected axes. These equations can be applied to obtain the principal stress ratios $P / T$ and $Q / T$ in terms of the stress ratios $\hat{r r} / T, \theta \theta / T$, and $\hat{r} \theta / T$. The angle of rotation of axes, namely, the angle between $r$ and $P$, is the difference between $\phi$, the angle from the direction of applied stress

\footnotetext{
${ }^{16} \mathrm{~K}$. Sezawa and G. Nishimura. Stresses in a stretched plate containing a circular insertion. Rep. Aeron. Research Inst., Tokyo Imp. Univ. 6, 25 (1931).

17 E. G. Coker and L. N. G. Filon. A Treatise on Photoelasticity, see page 115 (Cambridge Univ. Press, 1931).

18 See footnote 17.

10 See page 116 of reference 17.

20 Wilfred E. Thibodeau and Archibald T. McPherson. Photoelastic properties of soft, rulcanized rubber. J. Research NBS 13, 887 (1934) RP751. Reprinted in Rubber Chem. Tech. 8, 183 (1935): William L. Holt and Archibald T. McPherson. Change of volume of rubber on stretching: Effects of time, elongation, and temperature. J. Research NBS 17, 657 (1936) RP936. Reprinted in Rubber Chem. Tech. 10, 412 (1937).

э1 E. G. Coker. Photoelasticity for engineers. Gen. Elec. Rev. 23, 873 (1920).
} 
to $P$, and $\theta$, the angle from the direction of applied stress to $r$. In this manner one finds:

and

$$
\begin{aligned}
& \frac{P}{T}=\frac{1}{2}\left[\frac{\hat{r}}{T}+\frac{\hat{\theta}}{T}+\frac{\hat{r r}-\hat{\theta \theta}}{T \cos 2(\phi-\theta)}\right] \\
& \frac{Q}{T}=\frac{1}{2}\left[\frac{\hat{r r}}{T}+\frac{\hat{\theta \theta}}{T}-\frac{\hat{r r}-\hat{\theta \theta}}{T \cos 2(\phi-\theta)}\right]
\end{aligned}
$$

$$
\tan 2(\phi-\theta)=\frac{2 \widehat{r \theta}}{\widehat{r}-\widehat{\theta \theta}}
$$

Equations 17 to 22 can then be used to obtain at any point the theoretical values of $P / T, Q / T$, and $\phi$ in terms of the coordinates $(r, \theta)$. The results are shown in tables 2,3 , and 4 in the columns headed $P_{t} / T, Q_{t} / T$, and $\phi_{t}$, and in graphic form in figures 3,4 , and 5 .

\section{DISCUSSION OF RESULTS}

As might be expected, both $P / T$ and $Q / T$ can be seen to have their largest values in the region of the circumference of the disk near the axis in the direction of the applied load. The largest value of $Q / T$, according to both theory and observation, is found at the intersection of the circumference and this axis. The maximum of $P / T$ along this axis, however, by both theory and experiment, is found displaced somewhat from this point in the direction away from the disk. The maximum of $P / T$ around the circumference is also displaced somewhat from this point. The maximum of $(P-Q) / T$ at the circumference is found roughly halfway around the circumference of the quadrant.

What is not so obviously expected is the vanishing of the $P / T$ and $Q / T$ values on the boundary of the disk. Near the axis perpendicular to the direction of applied load the $P / T$ values become negative, thereby indicating a slight compression. $Q / T$ also has both positive and negative values, changing sign on the circumference about midway around the quadrant.

The maximum value of $P$ is about 50 percent greater than $T$, the applied tensile stress, while the value of $Q$ attains a maximum of the order of two-thirds of the applied tension.

Errors in the observed values, where they occur, may be ascribed to one or more of the following factors: (a) Errors inherent in the method used-(1) the admitted limitations of precision inherent in photoelastic determinations; (2) the difficulties of a graphic method of attack; and (3) the necessity of an extensive graphic integration to obtain some single points; (b) Errors entering into this particular investigation-(1) The difficulty of making photoelastic observations at the interface between the disk and the soft rubber, with the consequent necessity of some degree of extrapolation in a region where the stress variation is rapid; (2) the use of a single mean thickness instead of the actual thickness at each observed point; and (3) the uncertainty arising from the fact that the first observed point was not at the edge of the model.

The theoretical treatment involved assumptions contrary to the facts of the actual case in the following respects: (1) The sheet was assumed to be infinite in extent in both directions; $(2)$ the deformations 
were assumed to be infinitesimal; and (3) the effect of the clamps at the ends was neglected.

In view of all these factors involving possible causes of discrepancy, only the first four of which may be regarded as defects of the photoelastic method, agreement between observed and theoretical values is considered very satisfactory on the whole. The qualitative characters of the variations are the same in all cases. The locations of all critical values are identical, with the exception of $Q / T$ in figure 5 . The quantitative agreement seems as satisfactory as could be expected, except for those values of $Q / T$ in figure 4, which were obtained as the difference of two nearly equal quantities, and except for $Q / T$ in figure 5. In the latter case, not only were the observations difficult, but as the circumference of the disk is approached, there is a very rapid variation of $Q / T$, as can be seen in figures 3 and 4 .

\section{CONCLUSIONS}

This work outlines a method of experimental procedure and demonstrates, by a reasonably close agreement with the theoretical treatment of a problem of complex stresses, that rubber can be employed as a model material in photoelastic work. Unlike most model materials it remains free from residual stresses, and, although it exhibits comparatively large deformations when stressed, it has such a large stress-optical coefficient that good results can be obtained with very small applied loads, less than one one-hundredth the size of those usually employed. Stress problems in rubber itself, for which theoretical treatment is often very difficult, can be readily solved in this manner. The application of results obtained with rubber models to cases involving steel and other engineering materials should be made with caution, however, on account of the difference in the values of Poisson's ratio.

Acknowledgment is made of the valuable assistance rendered by Dr. H. B. Maris and Mr. R. B. Carleton of the U. S. Naval Research Laboratory, Bellevue, D. C., in providing for the use of their unusually fine equipment in this work.

Washington, December 28, 1937. 